\title{
Development of Evaluation Function for Openability of Opening Diameter of Aluminum Beverage Bottles and Its Application to Optimum Design
}

\author{
Takanori Chihara ${ }^{1}$ \\ Tokyo Metropolitan University, 6-6, Asahigaoka, Hino, Tokyo, 191-0065, Japan \\ Koetsu Yamazaki ${ }^{2}$ \\ Kanazawa University, Kakuma-machi, Kanazawa, Ishikawa, 920-1192,Japan \\ Saiki Sobajima ${ }^{3}$ \\ Kanazawa University, Kakuma-machi, Kanazawa, Ishikawa, 920-1192,Japan \\ and \\ Jing $\operatorname{Han}^{4}$ \\ Universal Can Corporation, Shizuoka, 1500, Suganuma, Oyama, Sunto-gun, Shizuoka,410-1392, Japan
}

\begin{abstract}
The present paper proposes an evaluation function of openability considering the transition of gripping postures, i.e. two-finger gripping and three-finger gripping, so as to evaluate opening ease of aluminum beverage bottles and to specify the rigorous dimension of the cap diameter that is best suited for consumers' satisfaction. First, we have investigated effects of cap diameter on the gripping postures. It is found that subjects tend to shift the gripping posture from two-finger gripping (gripping with thumb and first finger) to threefinger gripping (gripping with thumb, first, and second finger) with increase of the opening diameter. An evaluation function is defined as a ratio between maximum torques that consumers can apply and a required torque for opening. In addition, the cap diameter is the only design variable, and the thumb length, which associates with the transition of the gripping postures and a maximum gripping force, is selected as a state variable in order to take individual differences into consideration. Moreover, an evaluation function is formulated considering the transition of the gripping postures of consumers. The function is composed by using the data obtained from measurement of the maximum gripping force, the maximum torque, and the thumb length, and then the function is optimized. The result of optimization has shown that the optimum opening diameter is obtained at $28.0 \mathrm{~mm}$ in case of the subjects.
\end{abstract}

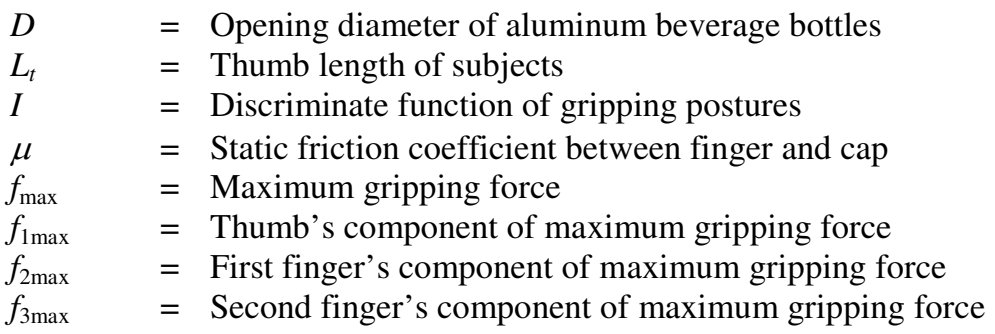

\footnotetext{
${ }^{1}$ Assistant Professor, Faculty of System Design.

${ }^{2}$ Professor, Collage of Science and Engineering, Fellow AIAA.

${ }^{3}$ Resarch Scholor, Collage of Science and Engineering.

${ }^{4}$ Manager, Technology and Development Department, AIAA member.
} 


$\begin{array}{ll}T_{\max } & =\text { Maximum torque } \\ T_{\text {open }} & =\text { Required torque } \\ F & =\text { Evaluation function of openability } \\ O_{1} & =\text { Burden ratio with two-finger gripping } \\ O_{2} & =\text { Burden ration with three-finger gripping } \\ c_{1} & =\text { Indicator of two-finger gripping } \\ c_{2} & =\text { Indicator of three-finger gripping } \\ \alpha & =\text { thumb-first finger angle } \\ \beta & =\text { thumb-second finger angle }\end{array}$

\title{
I. Introduction
}

\begin{abstract}
A luminum beverage bottles with screw tops have been launched in the Japanese market in recent years to meet the modern-day drinking habits of consumers. These can be repeatedly resealed, and are designed to recycle many times better than the resealable PET bottles. Therefore, aluminum beverage bottles are paid attention in the Japanese market because of the point of view on reduction of environmental burdens. The following features are required for beverage containers such as aluminum beverage bottles: improved shelf life (the length of time that packaged food can be stored), sealing performance, price, and so on. In addition to the above-mentioned basic features, universal designs based on ergonomics have been required while developing beverage containers and are expected to enhance consumers' convenience. For example, Han et al. have investigated that the relationship between tab ring shape and the feelings in the fingertip when opening aluminum beverage cans; and they have concluded that the tab with a larger contact area with finger is better ${ }^{1}$.

Openability of the bottle caps, which is one of a demand of aluminum beverage bottles for consumers' satisfaction, has been studied by some researchers ${ }^{2-7}$. However, these researchers have not determined the optimum opening diameter that is easy to open. Additionally, the difference of gripping postures has not been considered, while it is possible that the difference affects openability of caps. Therefore, to evaluate openability more precisely and determine the optimum opening diameter for openability, we derive an evaluation method for openability considering the difference of gripping postures.

In this study, first, effects of the cap diameter and hand dimensions on gripping postures is investigated. Next, a physical characteristic that represents openability is decided, and then an evaluation function of openability, which is formulated considering the difference of gripping postures and the individual differences, is defined. The response surface of the evaluation function is composed by using Radial Basis Function Network (RBFN). Then, the evaluation function is optimized to determine an optimum opening diameter that is easiest to open.
\end{abstract}

\section{Survey on Transition of Gripping Postures}

\section{A. Method}

A survey on gripping postures was carried out in order to investigate the effects of the cap diameter and the hand dimensions of subjects on the gripping postures. Subjects were 12 Japanese university students ( 8 males and 4 females), and all of them are healthy and righthanded. All subjects were seated during the experiment, and were required to twist the cap of the experimental bottles. The gripping postures of all subjects have been recorded using digital camera. As shown in Fig. 1, five kinds of experimental bottles with opening diameter of $28,33,38,43$ and $48 \mathrm{~mm}$ were used, and all of experimental bottles have been opened. In addition, we have also measured the hand dimensions of the subjects as shown in Fig. 2, because we have predicted that the

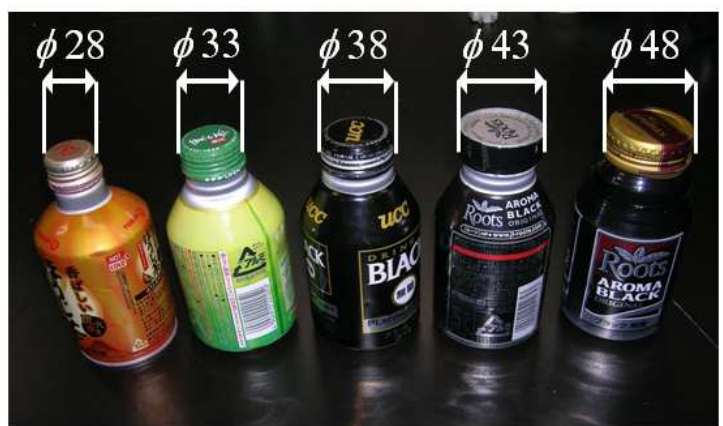

Figure 1. Five kinds of experimental bottle samples dimensions would affect the gripping posture. 


\section{B. Results and Discussions}

Three kinds of gripping postures shown in Fig. 3 have been found from the results of the experiment. Fig. 3 a) is clipping the cap with thumb and first finger (hereafter referred to as "two-finger gripping"), and Fig.3 b) is clipping the cap with thumb and second finger, and placing first finger (hereafter referred to as "three-finger gripping"). In addition, Fig. 3 c) is gripping with thumb and first finger in the inverse direction (hereafter referred to as "inverse gripping"). Among the 12 subjects, only two subjects gripped with the inverse gripping, and the other subjects gripped with two-fingers gripping and three-finger gripping; hence, we eliminate the data of the two subjects who gripped with the inverse gripping, and noted the two gripping postures hereafter.

Figure 4 represents the transition of gripping posture associated with the increase of the cap diameter. From Fig.4, it is clear that the relative frequency of the two-finger gripping decreases and that of the three-finger gripping increases with increase of opening diameter, and the order of the two relative frequency replace each other at $38 \mathrm{~mm}$. Thus, the gripping posture of the subjects transits from two-finger to three-finger gripping associated with increase of the opening diameter.

Transition diameter is defined as a diameter at which participant begins to grip with the three-finger gripping; here, we assume that the participant that grips all of the five opening diameter with the two-finger gripping begins to grip with the three-finger gripping at $53 \mathrm{~mm}$. Table 1 shows the correlation coefficients between the transition diameter and each dimensions of hand. In addition, Figure 5 illustrates a relationship between the transition diameter and the thumb length. From Table 1, all of hand dimensions are correlated with the transition diameter at a $1 \%$ significance level. Among the dimensions, the thumb length has the highest correlation coefficient $(r=0.837)$. In addition, as described later, the thumb length is also correlated with the maximum gripping force; therefore, we use the thumb length as a representative variable that represents individual differences in this paper. Moreover, we assume that the transition diameter is expressed by the linear function of the thumb length, and the discriminate function of gripping postures $I$ is given as a function of the opening diameter $D$ and the thumb length $L_{t}$ as follows;

$$
I\left(D, L_{t}\right)=D-1.54 L_{t}+51.6
$$

Here, gripping posture is the two-finger gripping when the function is less or equal to zero $(I(D, a) \leq 0)$, while the three-finger gripping when the function is greater than zero $(I(D, a)>0)$.

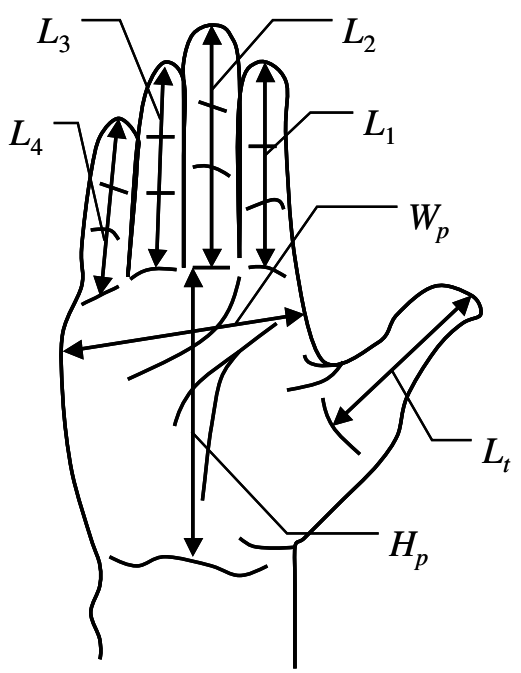

Figure 2. Anthropometric dimensions of hand

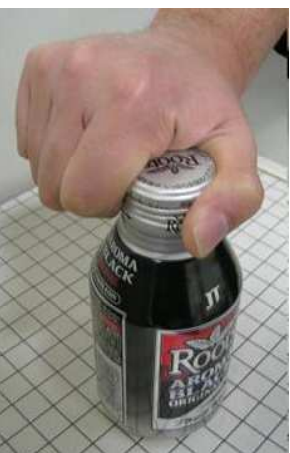

a) Two-finger

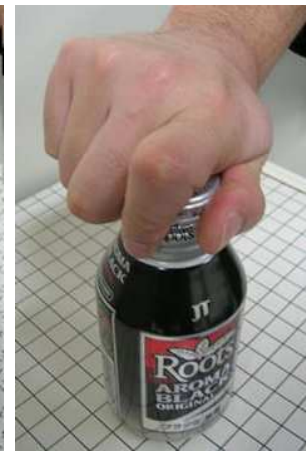

b) Three-finger

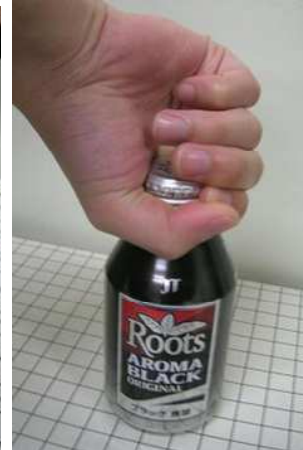

c) Inverse
Figure 3. Gripping postures

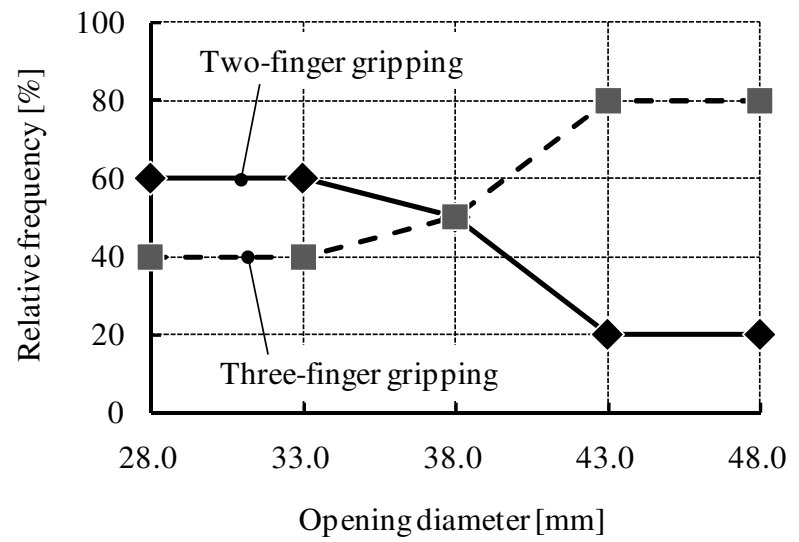

Figure 4. Transition of gripping posture 


\section{Evaluation Function of Openability}

\section{A. Definition of Evaluation Function of Open- ability}

In general, consumers grip the screw cap of the bottle with their dominant hand and grip the bottle body with their non-dominant other hand, when they open the screw cap. After the action, they twist their hands to open the screw cap. The torque required to open the screw cap of the bottle is equal to the torque that the consumers apply to the bottle body when they twist their hands. Because the diameter of the screw cap is smaller than that of the bottle body, the twisting torque required to open the cap is determined by the gripping force of screw top. Therefore, we focus on the gripping force of consumers so as to evaluate the opening diameter for consumers' satisfaction.

It is considered that opening ease is determined by the following factors: smaller required torque to open the cap and larger torque that consumers apply. However, the smaller required torque does not always give the opening ease, similarly, the larger torque applied by the consumers does not always give the pening ease. Therefore, we need to evaluate the required torque to open the cap (hereafter referred to as "required torque $T_{\text {open }}$ ") and the maximum torque that consumers can apply (hereafter referred to as "maximum torque $T_{\max }$ ") simultaneously. Thus, we use a ratio of the required torque to the maximum torque (hereafter referred to as "burden ratio $T_{\text {open }} / T_{\max }$ ") as an indicator of openability. In other words, we assume that the smaller burden ratio makes it easier to open the cap.

When the maximum gripping force that consumer can apply is defined as total applied force of all fingers in the radial direction of cap (hereafter referred to as "maximum gripping force $f_{\max }$ "), the maximum torque is given as follows;

$$
T_{\text {max }}=\frac{1}{2} \mu D f_{\max }
$$

where $\mu$ and $D$ denote the static friction coefficient between finger and the cap, and the opening diameter of the cap. Hence, the burden ratio is translated as follows:

$$
\frac{T_{\text {open }}}{T_{\max }}=\frac{2 T_{\text {open }}}{\mu D f_{\max }}
$$

Here, we apply a theoretical equation of required torque derived by Itoh et al. as the required torque ${ }^{8}$. The openability of aluminum beverage bottle is evaluated by the assumption that the smaller the burden ratio is, the more consumers feel easy to open the cap.

\section{B. Formulation of Evaluation Function of Openability}

We define the evaluation function of openability by considering the following two variables: the bottle opening diameter, and selected representative hand dimension. Between two variables, the bottle opening diameter is the only design variable, and the hand dimension is a state variable that fluctuates in a confined range. Hence, we must define the evaluation function as a function that evaluates the opening diameter in a given range of state variable. Therefore, we define the evaluation function as follows;

$$
F(D)=\int_{L_{\min }}^{L_{\max }}\left[\left\{O_{1}\left(D, L_{t}\right) \cdot c_{1}+O_{2}\left(D, L_{t}\right) \cdot c_{2}\right\} P_{L t}\left(L_{t}\right)\right] d L_{t}
$$

where index numbers 1 and 2 represent the two-finger gripping and the three-finger gripping. $a_{\min }$ and $a_{\max }$ are the 
minimum and maximum bounds of the hand dimension. $P_{L t}\left(L_{t}\right)$ is a weight function based on the distribution of the hand dimension ${ }^{9}$. In addition, $c_{1}$ and $c_{2}$ denote the identifier of the gripping postures, and they are given by the following equations;

$$
\begin{aligned}
& c_{1}= \begin{cases}1 & I\left(D, L_{t}\right) \leq 0 \\
0 & I\left(D, L_{t}\right)>0\end{cases} \\
& c_{2}= \begin{cases}1 & I\left(D, L_{t}\right)>0 \\
0 & I\left(D, L_{t}\right) \leq 0\end{cases}
\end{aligned}
$$

$O_{1}(D, a)$ and $O_{2}(D, a)$ are the burden ratio of the two-finger and three-finger gripping; and they are the response surface of the output predicted using the RBFN. The detailed procedure for constructing a response surface using the RBFN may be reffered to the references ${ }^{10}$. The smaller the value of $F(D)$, the lower the burden of opening is and consumers feel easy to open.

\section{Measurement of Maximum Gripping Force and Torque}

\section{A. Method}

We have measured the maximum gripping force when subjects grip and twist the cap. The contact area between the cap and fingers in case of the twofinger gripping may be smaller than in case of the three-finger gripping; therefore, it is possible that apparent friction coefficient between the cap and fingers changes depending on the gripping posture. Hence, we have also measured the maximum torque in addition to the maximum gripping

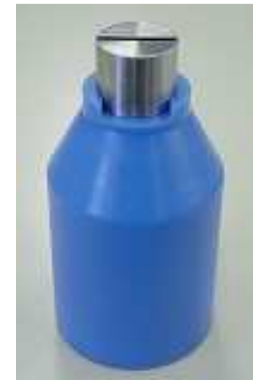

a) $28 \mathrm{~mm}$

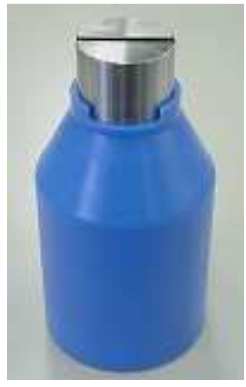

b) $33 \mathrm{~mm}$

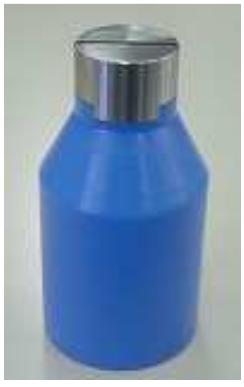

c) $38 \mathrm{~mm}$

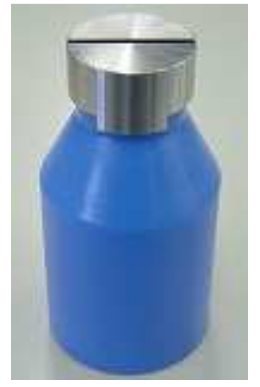

d) $43 \mathrm{~mm}$

Figure 6. Four kinds of bottle opening for measurement device force, and considered the effects of the gripping posture on the apparent friction coefficient.

We have performed an experiment in which subjects are 10 Japanese university students that are the same as the aforementioned survey excepting who have gripped with the inverse gripping. As shown in Fig. 6, four kinds of experimental bottles with opening diameter of 28, 33, 38, and $43 \mathrm{~mm}$ have been used, and each bottle opening has had a force sensor (9132B, KISTLER Co., Ltd.) inside of it. The normal forces in the radial direction of the cap have been measured by the force sensor, and have been kept computerized records through amplifier (5011B, KISTLER Co., Ltd.) and analog-digital converter (EC-2386, ELMEC Co., Ltd.). Simultaneously, the maximum torques have been measured by a torque meter (TNX-5, NIDEC-SHIMPO Co., Ltd.). The bottle opening devices have been fixed on a plastic bottle body, and the bottle opening devises do not rotate.

All subjects have been seated during the experiment, and have been required to twist the cap of the experimental bottles as much as possible for 2.0 seconds. In addition, they have been required to grip the cap with their right hand, and with the two-finger and three-finger gripping. The experiment has been repeated two times; hence, the number of trials of one subject is 16 times in total(i.e. 2 gripping postures, 4 experimental bottle openings, and 2 sets). Moreover, 10 minutes rest has been taken between each trial of the experiment, and measuring sequence has been randomized to erase the effect of order.

\section{B. Procedure for Calculating \\ Maximum Gripping Force}

The maximum gripping force of the two-finger gripping is calculated by doubling the thumb's component of the maximum gripping force (see Fig.7 a)).

In case of three-finger gripping, because the gripping force can be measured in only one direction by the force sensor, the thumb's component of the maximum gripping force has been

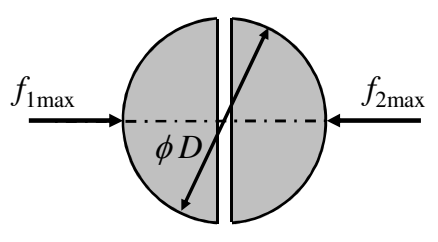

a) Two-finger gripping

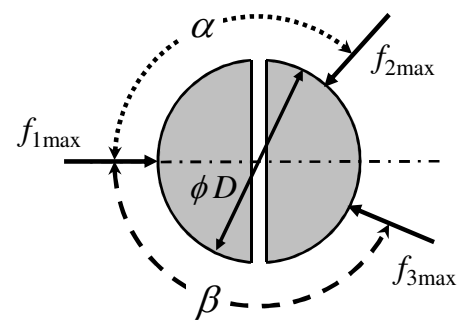

b) Three-finger gripping

Figure 7. Finger positions for equilibrium of force 
measured by placing the middle of proximal phalanx of thumb as shown in Fig. 8. In addition, the position and angles of fingers have been measured by using ink and paper as shown in Fig.9. Then, we have obtained the maximum gripping force by equilibrium of force (see Fig.7 b)). The equilibrium equations are given by

$$
\begin{gathered}
f_{\text {max }}=\left|f_{1 \text { max }}\right|+\left|f_{2 \max }\right|+\left|f_{3 \max }\right| \\
f_{1 \text { max }}+f_{2 \text { max }} \cos \alpha+f_{3 \max } \cos \beta=0 \\
f_{2 \max } \sin \alpha=f_{3 \max } \sin \beta
\end{gathered}
$$

where $f_{1 \max }, f_{2 \max }$, and $f_{3 \max }$ are thumb's, first finger's, and second finger's components of the maximum gripping force, respectively. In addition, $\alpha$ and $\beta$ denote the angles between the thumb and the first finger, and the thumb and the second finger.
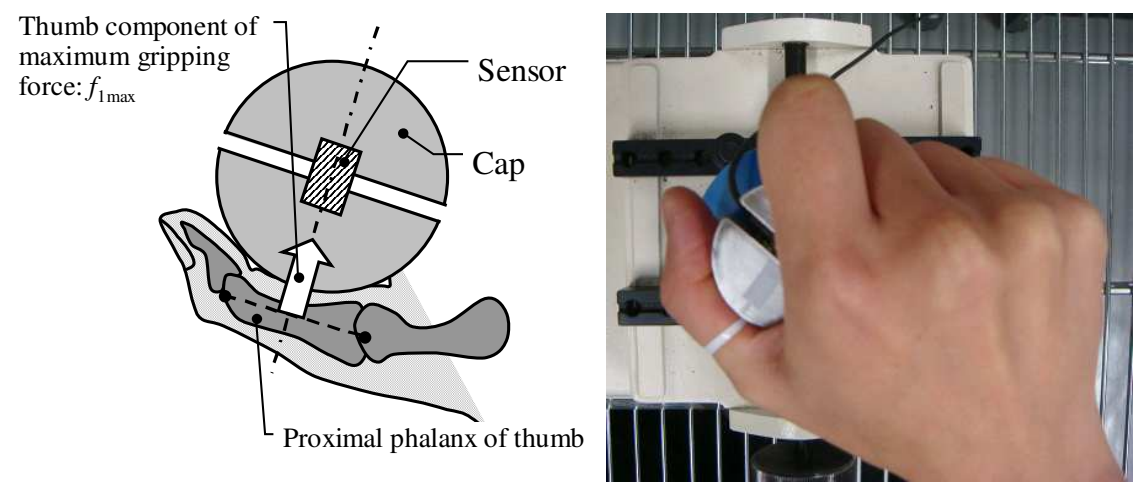

Figure 8. Measuring method for thumb component of maximum gripping force

\section{Results and Discussions}

Table 2 shows the correlation coefficients between the maximum gripping forces and each hand dimensions that have been measured in the aforementioned survey. It is found that the thumb length has relatively high correlation coefficient in case of twofinger gripping, and the highest correlation coefficient in case of three-finger gripping. As mentioned before, the thumb length is correlated with the transition diameter in addition to the maximum gripping force; therefore, we use the thumb length as a representative variable that represents individual differences.

The average values of the maximum gripping force and maximum torque are shown in Figs. 10 and 11. Moreover, Figure 12 shows the apparent friction coefficients that have been calculated by using the maximum gripping force and torque. The curves shown in Fig. 12 approximate the apparent friction coefficients by quadratic functions of opening diameters. In Fig. 10, the maximum gripping force of the two-finger gripping is higher than

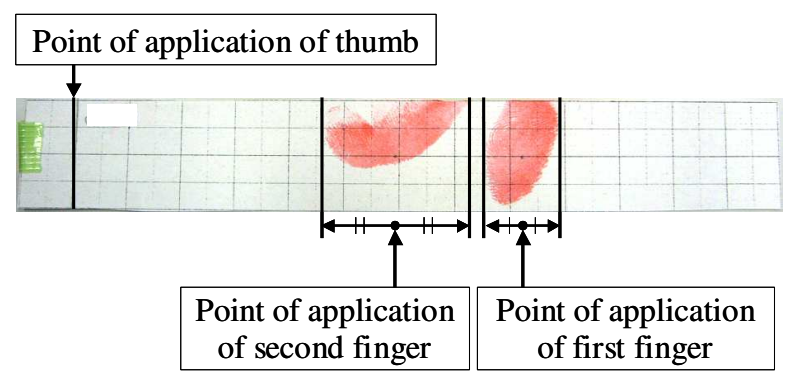

Figure 9. Example of contact area on first and second finger

Table 2. Correlation coefficient between maximum gripping force and anthropometric dimensions of hand

\begin{tabular}{c|c|c}
\hline Dimensions of hand & $\begin{array}{c}\text { Two fingers } \\
\text { gripping }\end{array}$ & $\begin{array}{c}\text { Three fingers } \\
\text { gripping }\end{array}$ \\
\hline Thumb length $L_{t}$ & $0.702^{* *}$ & $0.320^{* *}$ \\
First finger length $L_{1}$ & $0.603^{* *}$ & $0.265^{*}$ \\
Second finger length $L_{2}$ & $0.659^{* *}$ & $0.231^{*}$ \\
Third finger length $L_{3}$ & $0.680^{* *}$ & $0.270^{*}$ \\
Fourth finger length $L_{4}$ & $0.574^{* *}$ & $0.280^{*}$ \\
Palm width $W_{p}$ & $0.593^{* *}$ & $0.277^{*}$ \\
Palm length $H_{\mathrm{p}}$ & $0.714^{* *}$ & $0.298^{* *}$ \\
\hline \multicolumn{2}{|c}{$* \mathrm{p}<0.05, * * \mathrm{p}<0.01$}
\end{tabular}
that of the three-finger gripping over the entire range of opening diameter. Thus, the subjects perhaps feel easier to exert their force on caps when they grip with the two-finger gripping than the three-finger gripping. From Fig. 11, it is found that the maximum torque of the 
two-finger gripping is higher than that of the three-finger gripping when the opening diameter is relatively small, while there is little difference of maximum torque between the two-finger and three-finger gripping when the opening diameter exceed about $38 \mathrm{~mm}$. This may be explained the fact that the apparent friction coefficient of the three-finger gripping is higher than that of two-finger gripping as shown in Fig. 12; because, perhaps the contact area between caps and three fingers (i.e. thumb, first, and second finger) is larger than two fingers (i.e. thumb and first finger). Further, the apparent friction coefficient, at first, increases with the increase of opening diameter until it becomes the maximum value, and then it decreases. The explanation for this is that the contact area between caps and fingers increases with the increase of opening diameter when the opening diameter is relatively small, while it decreases when the opening diameter is relatively large; because, the contact location of fingers transit from the face to the tip of fingers with the increase of opening diameter. In addition, from the approximated curve shown in Fig. 12, the maximum apparent friction coefficient is obtained at about $35 \mathrm{~mm}$ in case of the two-finger gripping, while about $39 \mathrm{~mm}$ in case of three-finger gripping.

From these results described above, we can conclude that the subjects grip the caps with the two-finger gripping that is easy to exert their force when the opening diameter is relatively small, and the apparent friction coefficient of two-finger gripping begins to decrease at about $35 \mathrm{~mm}$; and then, they transit their gripping posture from the two-finger to the the threefinger gripping so as to maintain the apparent friction coefficient and the maximum torque.

\section{Results of Optimization and Discussions}

We have formulated Eq.(4) using the data obtained from the measurement of the maximum gripping force; then, we minimized the value ofEq.(4). In addition, the apparent friction coefficients shown in Fig. 12 have set on each opening diameter and gripping posture. The upper and lower bounds of the design and state variable are given as

$$
\begin{aligned}
& 28.0 \leq D \leq 43.0 \\
& 51.4 \leq L_{t} \leq 67.0
\end{aligned}
$$

The two kinds of evaluation functions of openability shown in Fig 13 are composed by using the results of the two-finger and the three-finger gripping, respectively. In other words, they are formulated without considering the transition of gripping postures. As shown

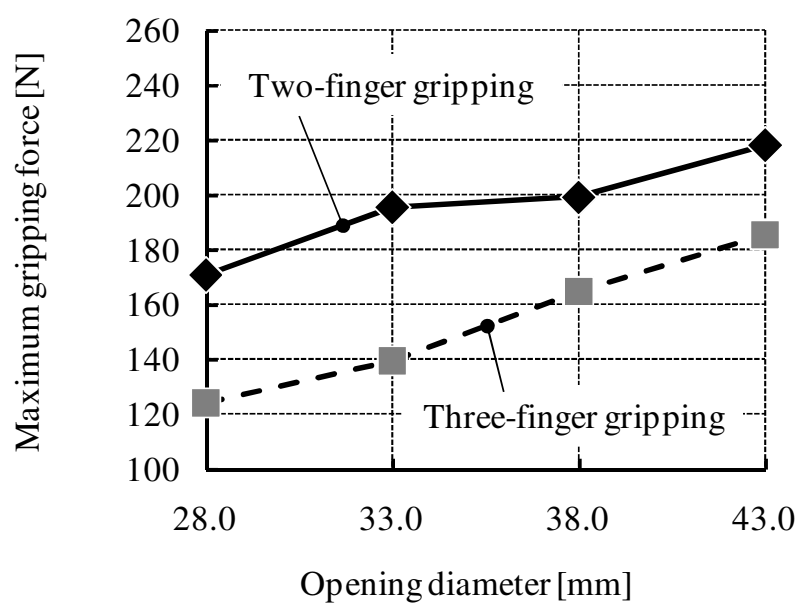

Figure 10. Average value of maximum gripping force

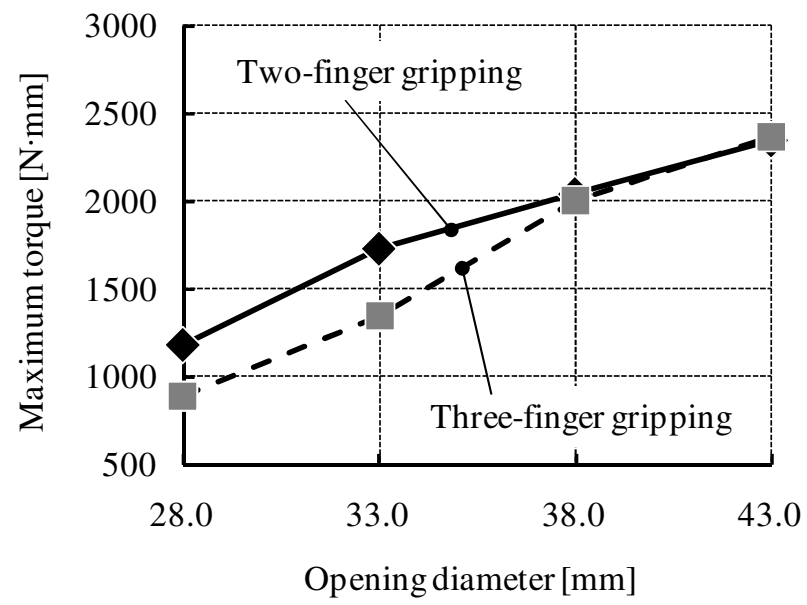

Figure 11. Average value of maximum torque

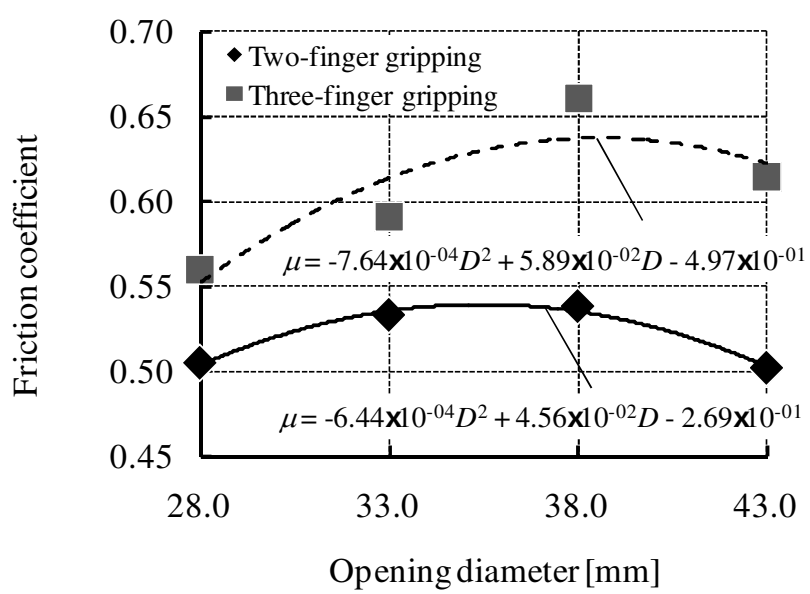

Figure 12. Average value of friction coefficient between finger skin and bottle caps 
in Fig. 13, the optimum of the evaluation function in the case of two-finger gripping is obtained at $28.0 \mathrm{~mm}$, while that in the case of three-finger gripping is obtained at $36.9 \mathrm{~mm}$. Hence, it is found that the optimum opening diameter on openability probably changes if the gripping posture changes. Further, the two evaluation functions intersected each other at about $40 \mathrm{~mm}$ in opening diameter, and the magnitudes of them are inverted. This is in agreement with the result that the relative frequency of the two-finger and three-finger gripping replaced each other at $38 \mathrm{~mm}$. Therefore, perhaps the subjects try to keep the burden ratio or the openability with the transition of the gripping posture.

Figure 14 shows the evaluation function that considers the transition of gripping postures. In addition, Figure 15 shows the average values of openability based on the subjective evaluation of the subjects when each subject grips each opening diameter with their gripping postures. That is, the gripping postures are the same as what the subjects have done in the survey described in chapter 3. The satisfaction scores have been collected on a scale of -3 (very difficult to open) to 3 (very easy to open); thus, the higher the satisfaction score is, the easier to open the cap and subjects probably feel comfortable. The error bars in Fig. 15 represent standard errors of the satisfaction scores.

From Fig. 14, the optimum of the evaluation function is obtained at $28 \mathrm{~mm}$. Hence, the burden ratio of the opening with a diameter of $28.0 \mathrm{~mm}$ is the lowest in the range of Eq.(13); but, it should be note that the optimum opening diameter, $28 \mathrm{~mm}$, is for this subjects. On the other hand, from Fig. 15 , the $38 \mathrm{~mm}$ has the highest average satisfaction score among the four kinds of opening diameters. However, there are no significant differences between the four satisfaction scores. This may be explained the fact that it is hard for the subjects to definitely feel the difference of openability; because they transit their gripping postures so as to maintain the maximum torque and the burden ratio. Therefore, it is difficult to decide the opening diameter for the openability based on the subjective evaluation of the subjects. The proposed evaluation function, however, can evaluate the opening diameter for openability quantitatively, because the function is based on the objective evaluation (i.e., burden ratio); thus, the $28 \mathrm{~mm}$ opening, which is the optimum of the proposed function, may be more reliable.

\section{Conclusions}

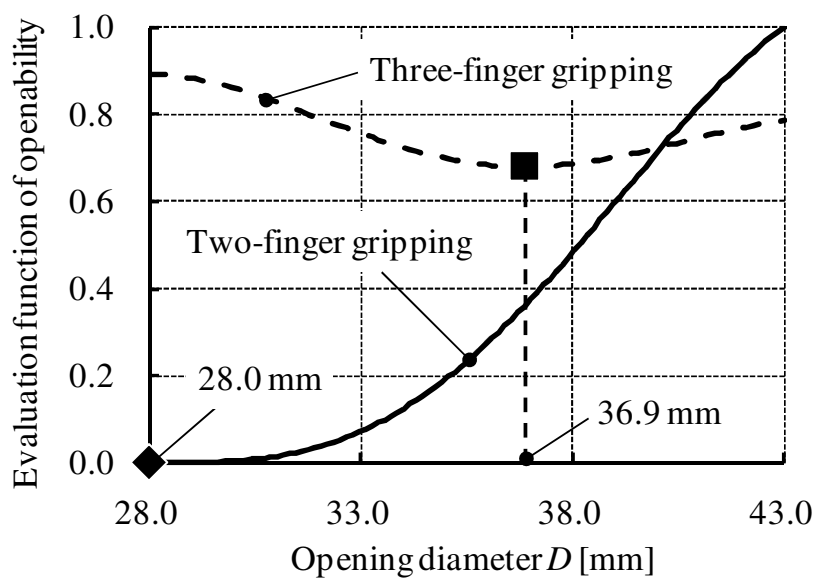

Figure 13. Evaluation function of openability on each

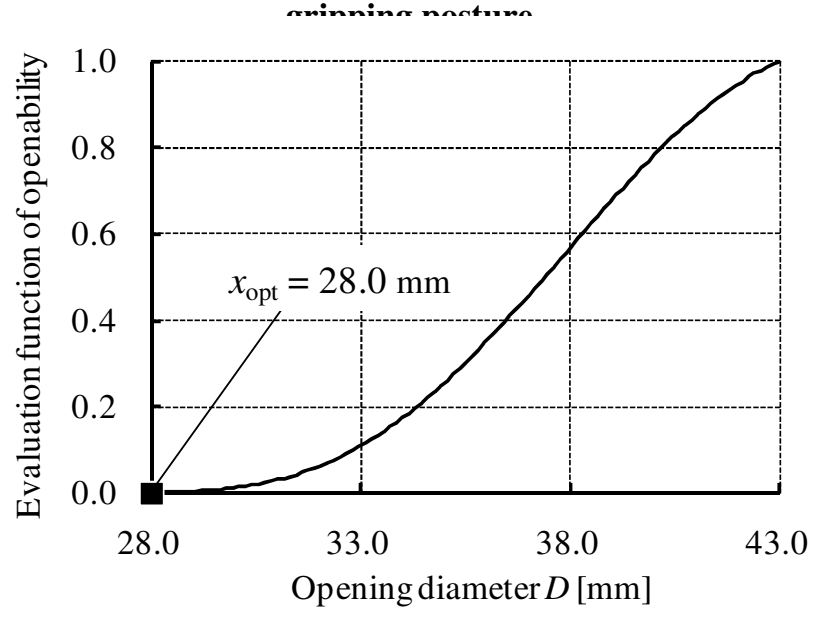

Figure 14. Evaluation function of openability

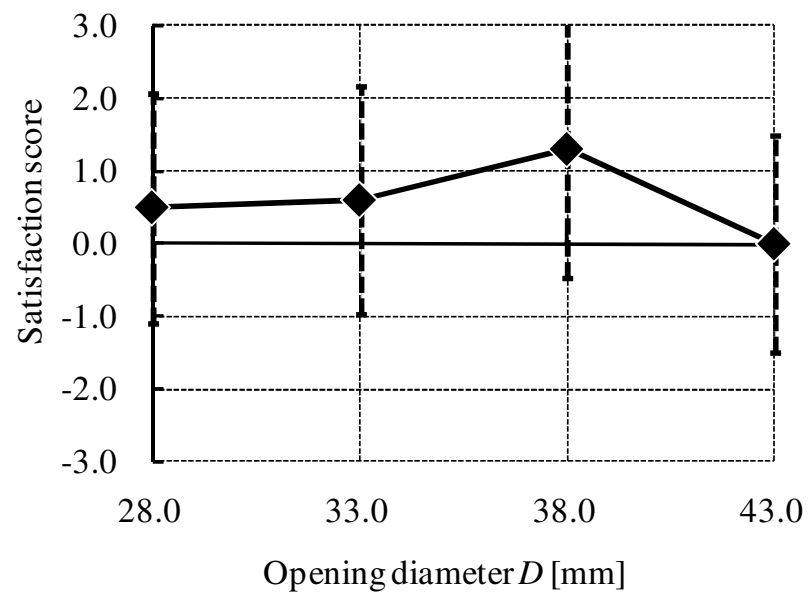

Figure 15. Average value of degree of openability

In this study, we have proposed an evaluation function of openability, which considers the transition of gripping postures and the individual differences, for determining the optimum opening diameter for opening ease. We have

8

American Institute of Aeronautics and Astronautics 
also optimized the evaluation function by performing experiments and have found that the burden ratio is lowest and subjects' comfort level is highest when the bottle opening diameter is $28.0 \mathrm{~mm}$. If a certain gripping posture is intended, especially the three-finger gripping in case of this study, the true optimum diameter for the openability may not be obtained. Therefore, the proposed evaluation function can determine the precise optimum diameter with considering the transition of gripping postures. In addition, we have also found that the optimal opening diameter depends on individual differences, i.e., the thumb length of consumers. Thus, the proposed evaluation function can be used to determine the optimum opening diameter of bottles that are to contain beverages targeted at a particular category of consumers.

\section{References}

${ }^{1}$ Han, J., Nishiyama, S., Yamazaki, K., Itoh, R., "Ergonomic design of beverage can lift tabs based on numerical evaluations of fingertip discomfort”, Applied Ergonomics, Vol.39, No.2, 2008, pp.150-157.

${ }^{2}$ Imrhan, Sheik N., Loo, Chong, "Torque capabilities of the elderly in opening screw top containers", Proc. of Human Factors and Ergonomics Society Annual Meeting, Vol.30, No.12, Human Factors and Ergonomics Society, Dayton, OH, 1986, pp.1167-1171.

${ }^{3}$ Crawford, Joanne O., Wanibe, E., Nayak, L., "The interaction between lid diameter, height and shape on wrist torque exertion in younger and older adults", Ergonomics, Vol.45, No.13, 2002, pp.922-933.

${ }^{4}$ Voorbij, A.I.M., Steenbekkers, L.P.A., "The twisting force of aged consumers when opening a jar", Applied Ergonomics, Vol.33, No.1, 2002, pp.105-109.

${ }^{5}$ Yoxall, A., Langley, J., Janson, R., Wearn, J., Manson, G., "The Use of Uncertainty Analysis for the Design of Container Closures", Packaging Technology and Science, Vol.19, No.6, 2006, pp.139-147.

${ }^{6}$ Lewis, R., Menardi, C., Yoxall, A., Langley, J., "Finger friction: Grip and opening packaging”, Wear, Vol.263, No.7-12, 2007, pp.1124-1132.

${ }^{7}$ Yoxall,A., Luxmoore, J., Rowson, J., Langley, J., Janson, R., "Size Does Matter: Further Studies in Hand-Pack Interaction Using Computer Simulation", Packaging Technology and Science, Vol.21, No.2, 2008, pp.61-72.

${ }^{8}$ Itoh, R., Yamazaki, K., Han, J., "A Study on Openability of an Aluminum Beverage Bottle”, Transactions of the Japan Society of Mechanical Engineers, Series C, Vol.74, No.738, 2008, pp.475-483. [in Japanese]

${ }^{9}$ National Institute of Bioscience and Human Technology, "Human body dimensions data for ergonomic design", Japan Publication Service, Tokyo, No.1996, pp.81-117.

${ }^{10}$ Orr, M.J.L, "Introduction to radial basis function networks", URL: http://anc.ed.ac.uk/rbf/rbf. html, [cited 1996]. 